\title{
TEVATRON ALIGNMENT ISSUES 2003-2004
}

\author{
J T Volk, J Annala, L Elementi, N Gelfand, K E Gollwitzer, J Greenwood, M Martens, C Moore, A \\ Nobrega, A D Russell, V Shiltsev, R Stefanski, T Sager, M J Syphers, G Wojcik \\ FNAL, Batavia, Ill. 60510, USA
}

\begin{abstract}
It was observed during the early part of Run II that dipole corrector currents in the Tevatron were changing over time. Measurement of the roll for dipoles and quadrupoles confirmed that there was a slow and systematic movement of the magnets from their ideal position. A simple system using a digital protractor and laptop computer was developed to allow roll measurements of all dipoles and quadrupoles. These measurements showed that many magnets in the Tevatron had rolled more than 1 milliradian. To aid in magnet alignment a new survey network was built in the Tevatron tunnel. This network is based on the use of free centering laser tracker. During the measurement of the network coordinates for all dipole, quadrupole and corrector magnets were obtained. This paper discusses roll measurement techniques and data, the old and new Tevatron alignment network.
\end{abstract}

\section{INTRODUCTION}

The large corrector magnet currents in RUN II indicated problems with alignment of Tevatron magnets. During the 3 week shutdown of January 2003 roll measurements of all 775 dipoles and 203 quadrupoles were made. The 1983 installation tolerance for magnet roll was $\pm 1 \mathrm{mr}$., and the actual measured RMS was $0.2 \mathrm{mr}$. Measurments indicated that some mangets had rolled by more than $8 \mathrm{mr}$ since initial alignment. A program was started during the next two shutdowns to un-roll all dipoles and quads. The aim was to achieve a roll tolerance of $\pm 1 \mathrm{mr}$. In addition a new survey network was constructed during the 2004 shutdown. This network superseeded the MURPHY Line [1] system that had been in use since 1983. The new network will allow for the use of free centering laser trackers for magnet alignment.

\section{EFFECT OF ROLLED DIPOLES ON ORBITS}

The effect of dipole rolls can be determined with a little algebra. The magnetic field initially is in the vertical direction;

$$
\mathbf{B}=\mathrm{B}_{\mathrm{y}} \mathbf{j}
$$

If the magnet is rolled by an angle $\theta$, the magnetic field seen by the beam is:

$$
\mathbf{B}=\mathrm{B}_{\mathrm{y}} \sin (\theta) \mathbf{i}+\mathrm{B}_{\mathrm{y}} \cos (\theta) \mathbf{j}
$$

Submitted May 2005

volk@fnal.gov

Work supported by the U.S. Department of Energy under contract No. DE-AC02-76CH03000
The change in the field is:

$$
\Delta(\mathbf{B})=\mathrm{B}_{\mathrm{y}} \sin (\theta) \mathbf{i}+\mathrm{B}_{\mathrm{y}}(1-\cos (\theta)) \mathbf{j}
$$

which for small $\theta$ is:

$$
\Delta(\mathbf{B})=\mathrm{B}_{\mathrm{y}} \theta \mathbf{i}+\mathrm{B}_{\mathrm{y}}\left(\theta^{2} / 2\right) \mathbf{j}
$$

In tracking codes such as Tevlat or MAD, [2] we treat $\Delta \mathbf{B}$ as producing a kick that modifies the nominal closed orbit produced by the field $\mathrm{B}$. The kicks corresponding to a change of $\Delta \mathbf{B}$ is:

$$
\begin{aligned}
\Delta \mathrm{x}^{\prime} & =\left(-\Delta \mathrm{B}_{\mathrm{y}} / \mathrm{B}\right)(\mathrm{B} \cdot \mathrm{l}) /([\mathrm{B} \rho]) \\
\Delta \mathrm{y}^{\prime} & =\left(+\Delta \mathrm{B}_{\mathrm{x}} / \mathrm{B}\right)(\mathrm{B} \cdot \mathrm{l}) /([\mathrm{B} \rho])
\end{aligned}
$$

Where $[\mathrm{B} \rho]$ is a measure of the momentum of the particles.

$$
[\mathrm{B} \rho](\mathrm{Tm})=3.3356 \cdot \mathrm{P}(\mathrm{GeV} / \mathrm{c})
$$

Then $(\mathrm{B} \cdot 1) /([\mathrm{B} \rho]$ is the bend angle in the dipoles which for the Tevatron is $8.12 \mathrm{mr}$. Thus:

and

$$
\Delta \mathrm{x}^{\prime}=-4.06 \cdot 10^{-3} \cdot \theta^{2}
$$

$$
\Delta \mathrm{y}^{\prime}=+8.12 \cdot 10^{-3} \cdot \theta
$$

Because the roll angle $\mathrm{T}$ is small, less than $10 \mathrm{mr}$, the value of $\Delta \mathrm{x}^{\prime}$ is small, less than $10^{-6} \mathrm{mr}$ and can be dropped. The change in the vertical closed orbit due to a kick of strength $\Delta \mathrm{y}^{\prime}$ is known to be:

Where

$$
\Delta y(s)=\Delta y^{\prime}\left(\beta(s)_{y} \beta_{y 0}\right)^{1 / 2} \cos (\psi-\pi v) / 2 \sin (\pi v)
$$

- $y(s)$ is the closed orbit distortion at a point a distance $\mathrm{s}$ from the position point of the kick.

- $\beta(s)_{y}$ is the value of the $\beta_{y}$ function at $s$

- $\beta_{\mathrm{y} 0}$ is the value of the $\beta_{\mathrm{y}}$ function at the position of the kick

- $v$ is the tune

- $\psi$ is the phase advance from the point of the kick to the point $\mathrm{s}$

In the Tevatron lattice the maximum value of $\beta$ in the arcs is $\approx 100 \mathrm{~m}$ and the current operating tune is $\approx 0.58$. Thus a $1 \mathrm{mr}$ roll can produce an orbit distortion as large as $\approx 0.3 \mathrm{~mm}$. The effect of the systematic rolls of the dipoles, produces a large distortion the vertical closed orbit. The measured rolls alone can generate a closed orbit distortion with excursions $>20 \mathrm{~mm}$ and in regions with large $\beta$ distortions larger than the physical aperture. 


\section{ROLL MEASUREMENTS}

When the Tevatron dipoles and quadruples were first manufactured field measurements were made at the Fermilab Magnet Test Facility. Four survey lugs were attached to the outside of each magnet two on either side of the upstream and two on either side of the downstream end of the magnet. The lugs were set such that the internal dipole field was vertical when the lugs were level with respect to gravity. The spacing across the magnet is $381 \mathrm{~mm}$, and the distance along the beam is $3404 \mathrm{~mm}$ for dipoles and $927 \mathrm{~mm}$ for normal quads. By measuring the angle of the lugs with respect to gravity the roll of the magnet can be calculated.

In the initial installation of the Tevatron the lugs were set level to with in $\pm 1 \mathrm{mr}$ tolerance. The lugs are also used to set the absolute elevation of the magnets around the ring.

The current system used to measure roll was derived from an earlier system developed by Hans Jostlein and Craig Moore. That system used a 25 year old Talyvel Clinometer mounted onto a fixture that rests on the fiducials of the magnet. The measurements taken were manually entered into a spreadsheet for later analysis.

The updated system uses a similar fixture with a Mitutoyo Digital Protractor, model 950-316. The interface uses an RS232C cable with a switch (model $10 \mathrm{P}-25 \mathrm{P}$ ) and the laptop's serial port.

Executable routines used to acquire data and interface with the operator were written using the graphical software system LabVIEW. An MS Excel spreadsheet of the magnet names and tunnel position to be surveyed is the reference database for the program.

When the program is started, the operator can begin collecting measurements from the beginning of the database, resume from his last known position or enter some other magnet location in the database.

Two measurements are taken at each end of the magnet and averaged to eliminate any systematic offset. The first measurement is taken with the fixture in one orientation; the fixture is rotated $180^{\circ}$ about the vertical axis for the second measurement. The program checks the reliability of the data and if the difference between the measurements is larger than $4 \mathrm{mrad}$, the operator is prompted to either accept the measurement or repeat it. The operator can also add comments after each measurement noting any special circumstances. A second limit of 25 mrad generates a red pop up window for the operator.

The collected data is saved in an MS Excel text file which contains the magnet name and position, time of the measurement, measured data, averages of the data, and automatically generated comments of the measurement along with the optional operator comments. The beginning of the file includes the name(s) of the operator(s) and date.

Using two such setups and two people for each setup all the dipoles and quadrupoles in the Tevatron could be measured in 3 days.

Figure 1 shows the rolls as a function of location as measured in 2003. There are large deviations in roll in A sector and in E sector. The consensus is that ground motion and rusting of stands caused the increase in roll in the 20 years since installation.

Roll Measurements October 2003 all magnets

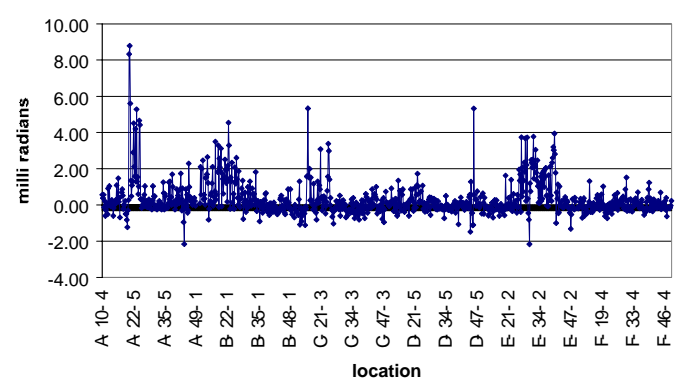

Figure 1 Roll measurements October 2003

Figure 2 shows the distribution of rolls as of November 2004 note change in vertical axis

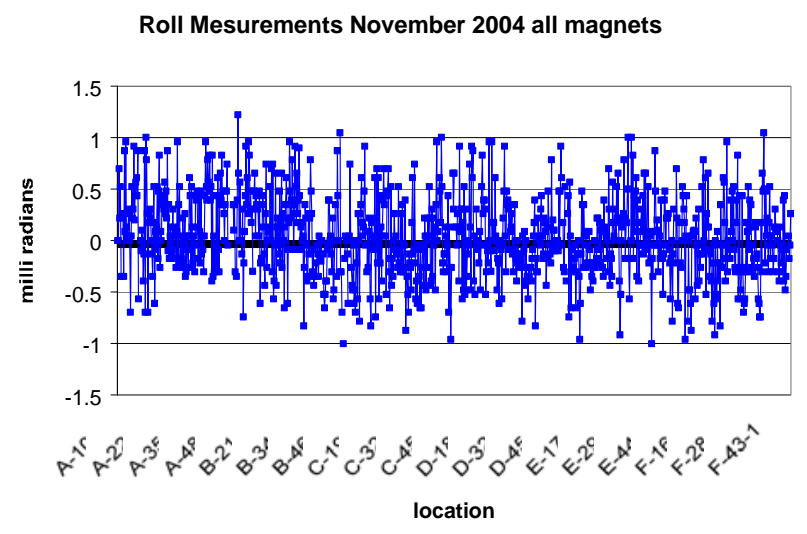

Figure 2 Roll measurements November 2004

All of the rolls are within the $\pm 1 \mathrm{mr}$ roll tolerance. Figure 3 shows the difference of the corrector currents around the ring. There is a significant decrease in the strength of the corrector currents after the roll correction. This indicates that there is a reduction in the orbit distortions due to rolled magnets around the ring. 


\section{TEVNET}

The original network for the Tevatron was developed by C. Thornton Murphy [1] in the early 1980s. The system consisted of 200 unconnected offset lines around the ring. These offset lines are referred to as Murphy lines. Each line is $29.6 \mathrm{~m}$ (97 feet) long and set such that the beam center line is $673.735 \mathrm{~mm}$ (26.525 inches) from beam center at each Tevatron quadrupole. The four dipoles that make up the half-cell all have different offsets from the Murphy line. The lines start half way between each halfcell and use brass floor plugs to mark the ends. Optical tooling is used to mechanically measure and move them into their designed position. The position of the Murphy line in the tunnel was determined by measurements from the Main Ring quadrupoles, there was no network outside of the tunnel. Elevations were provided by a series of benchmarks called tie rods. Three-wire level runs around the ring established the relative elevations of the tie rods.

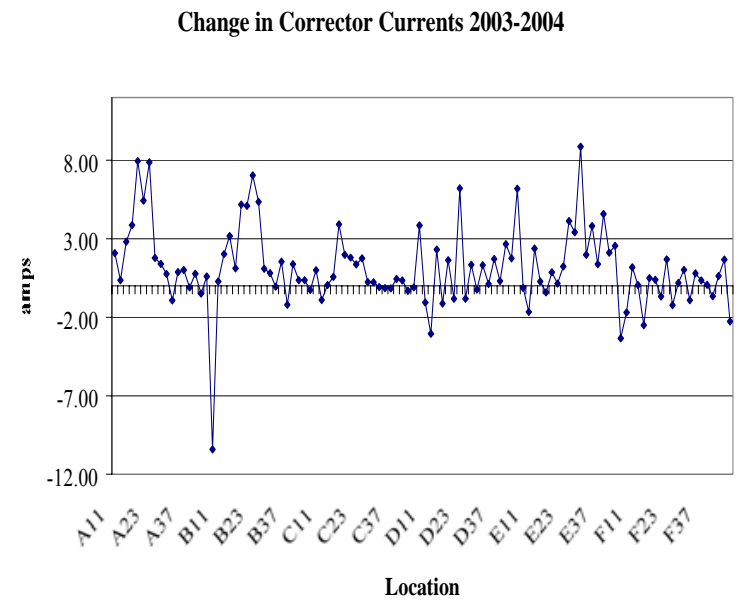

Figure 3 Corrector Current difference

To improve alignment tolerances a new network was constructed in the fall of 2003. This network consisted of braced polygons between new floor monuments, site riser drop points, pass points, and the tie rods around the tunnel. Over 1,827 such points were used in the tunnel. This system will allow for the use of free centering laser trackers to determine the position of all devices in the Tevatron.

In addition a surface control network of both horizontal and vertical networks defined by concrete monuments around the Tevatron and Main Injector rings were used Figure 4. The surface network was tied into the tunnel network at 14 points (site risers). The surface network used GPS baselines and Mekometer distance measurements. Vertical measurements were made with digital levels.

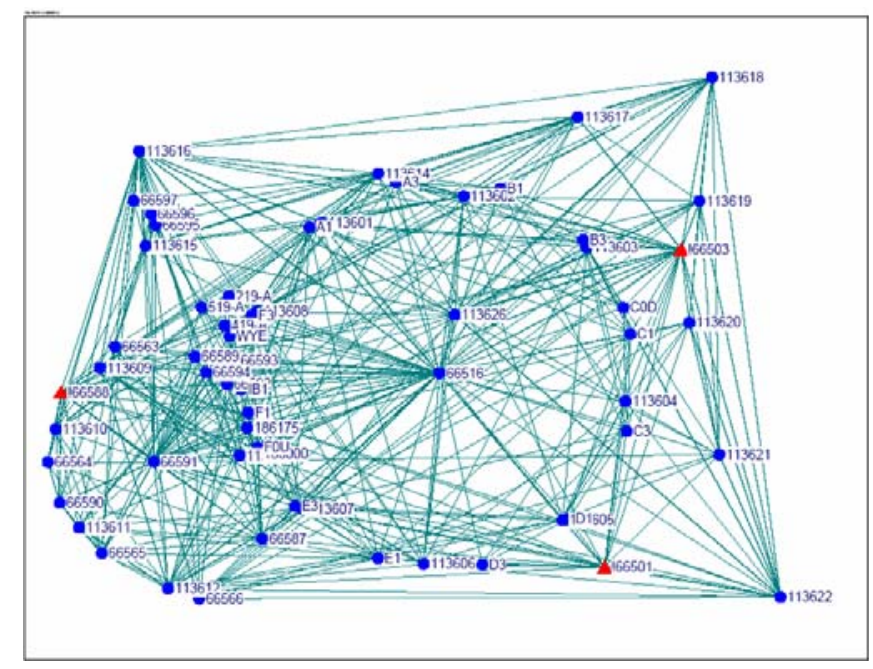

Figure 4 above ground survey network

During the observation of the Tev Net, laser trackers were used to measure all of the tunnel monuments and all quadrupoles and dipoles in the Tevatron. All of these data were adjusted using the least square method to provide the final coordinates for all points measured in the Tev Net. These data were then combined with the roll measurements to provide a complete set of coordinates for all quadrupoles and dipoles in the Tevatron.

Having coordinates of all dipoles and quadrupoles in the Tevatron allowed for comparison between the data and a model of the Tevatron used in Tevlat. The measured coordinates were translated into a ring centered flat plane system. Deviations between the data and the model were also compared with corrector magnet currents to identify magnets that were not on the ideal orbit for the Tevatron. From this a list of misaligned magnets was made along with necessary corrections.

\section{REFERENCES}

[1] How the Tevatron was Aligned C Thornton Murphy http://beamdocs.fnal.gov/cgi-

bin/public/DocDB/ListByAuthor?authorid=75

[2] Tevlat A Russell private communication and MAD Methodical Accelerator Design CERN-SL90-13-AP-rev-3 or http://hansg.home.cern.ch/hansg/mad/mad8/user/mad.ht $\mathrm{ml}$ 
